\title{
BERLINER BEITRÄGE ZUR NAMENFORSCHUNG
}

Herausgegeben von

K. Gutschmidt und H. Schmidt

im Auftrag des

Geisteswissenschaftlichen Zentrums Geschichte und Kultur Ostmitteleuropas e. V.

Band 12

BRANDENBURGISCHES NAMENBUCH

Teil 11

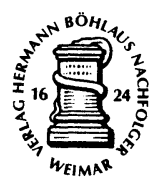




\title{
BRANDENBURGISCHES NAMENBUCH
}

\author{
Teil 11 \\ DIE ORTSNAMEN \\ DES LANDES RUPPIN
}

Von

ELŻBIETA FOSTER

mit einem siedlungsgeschichtlichen Beitrag von Christa Plate 
Die Deutsche Bibliothek - CIP-Einheitsaufnahme

Die Ortsnamen des Landes Ruppin / von Elżbieta Foster.

Mit einem siedlungsgeschichtlichen Beitr. von Christa Plate.-

Weimar : Verlag Hermann Böhlaus Nachfolger Weimar, 1998

(Brandenburgisches Namenbuch ; Teil 11)

(Berliner Beiträge zur Namenforschung ; Bd. 12)

ISBN 978-3-7400-1061-4

ISBN 978-3-7400-1061-4

ISBN 978-3-476-03124-2 (eBook)

DOI 10.1007/978-3-476-03124-2

Dieses Werk einschließlich aller seiner Teile ist urheberrechtlich geschützt. Jede Verwertung außerhalb der engen Grenzen des Urheberrechtsgesetzes ist ohne Zustimmung des Verlages unzulässig und strafbar. Das gilt insbesondere für Vervielfältigungen, Übersetzungen, Mikroverfilmungen und die Einspeicherung und Verarbeitung in elektronischen Systemen.

(C) 1998 Springer-Verlag GmbH Deutschland

Ursprünglich erschienen bei Verlag Hermann Böhlaus Nachfolger Weimar GmbH \& Co. 1998 


\section{VORWORT}

Der vorliegende Band ist eine Fortsetzung des Brandenburgischen Namenbuches. Das Arbeitsgebiet wird in Übereinstimmung mit dem Historischen Ortslexikon für Brandenburg in den Kreisgrenzen im Jahre 1900 festgelegt. Die Gesamtkonzeption folgt im Prinzip den bereits erschienenen Bänden. Bei den vorgenommenen Veränderungen wird vor allem der Zuwachs an theoretischen und methodischen Erkenntnissen bei der Namenkunde berücksichtigt und dabei nicht der Rahmen der für den Autor eingeräumten Interpretationsfreiheiten überschritten.

Die Arbeit an diesem Band wurde im Zentralinstitut für Sprachwissenschaft der Akademie der Wissenschaften der Deutschen Demokratischen Republik begonnen. Seit der Übernahme der Arbeitsgruppe Namenforschung vom Forschungsschwerpunkt Geschichte und Kultur Ostmitteleuropas der Förderungsgesellschaft Wissenschaftliche Neuvorhaben mbH im Jahre 1992 konnten die Untersuchungen auf diesem Gebiet weitergeführt und zum Abschluß gebracht werden.

Die siedlungsgeschichtliche Einleitung zu diesem Band wurde von Christa Plate verfaßt, der dafür mein herzlicher Dank gebührt. Ferner danke ich meinen Kollegen Frau Dr. Sophie Wauer für eine umfangreiche Beratung und Unterstützung, Frau Dr. Cornelia Willich und Herrn Dr. Reinhard Fischer für die Durchsicht des Manuskriptes sowie Herrn Dr. Klaus Müller für manchen Hinweis. Mein Dank gilt auch dem Leiter des Brandenburg-Berlinischen Wörterbuches Herrn Dr. Joachim Wiese und seinem Team, die mir Einblick in das lexikalische Material des Archives ermöglichten. Schließlich möchte ich auch den Herausgebern für kritische Bemerkungen und Anregungen danken.

Das Erscheinen dieses Bandes des Brandenburgischen Namenbuches ist dem Geisteswissenschaftlichen Zentrum für Geschichte und Kultur Ostmitteleuropas e. V. und der großzügigen Unterstützung seines Direktors Herrn Prof. Dr. Winfried Eberhard zu verdanken. 


\section{INHALT}

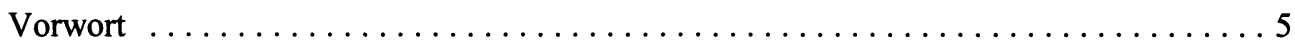

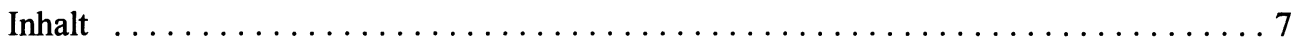

I. Die Besiedlungsgeschichte des Landes Ruppin (von Christa Plate) . . . . . . . . 9

A. Die Ur- und Frühgeschichte $\ldots \ldots \ldots \ldots \ldots \ldots \ldots \ldots \ldots$

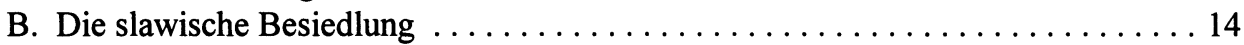

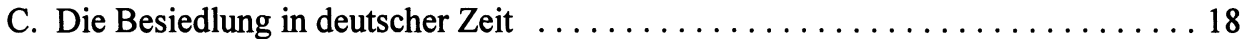

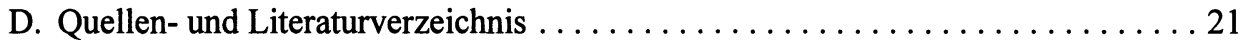

Tabelle: Slawische und frühdeutsche Befestigungen Ruppins $\ldots \ldots \ldots \ldots \ldots 24$

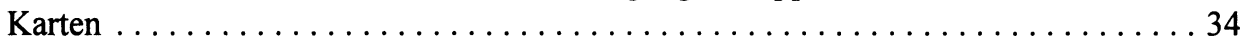

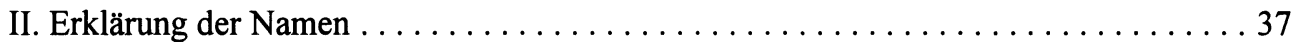

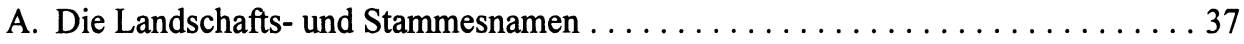

B. Die Namen der Städte, Dörfer, Ortsteile, Wohnplätze und Wüstungen . . . . . 40

C. Die Namen von ehemaligen oder bestehenden kleineren Siedlungen, . . . . . 131 Förstereien, Mühlen und anderen Einrichtungen

D. Gewässernamen . . . . . . . . . . . . . . . . . . . . . . . . . . . . . . . . . . . . . . 144

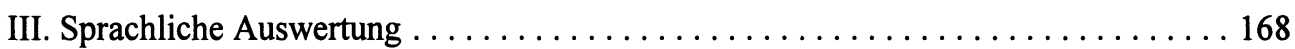

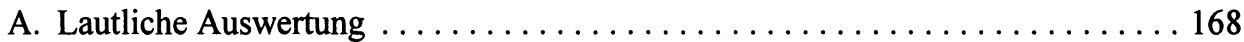

1. Die Vertreung der urslawischen Laute im Altpolabischen $\ldots \ldots \ldots \ldots \ldots 8$ und deren Übernahme ins Deutsche

a) Vokale und Halbvokale . . . . . . . . . . . . . . . . . . 169

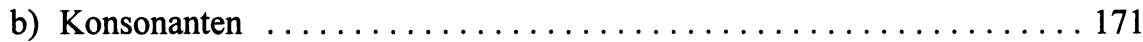

c) Kombinatorischer Lautwandel . . . . . . . . . . . . . . . . 173

d) Die Betonung der slawischen Ortsnamen $\ldots \ldots \ldots \ldots \ldots \ldots \ldots \ldots 174$

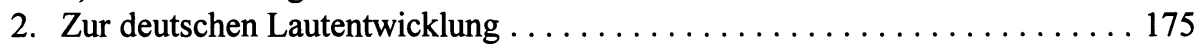

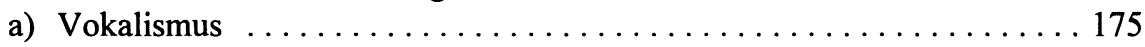

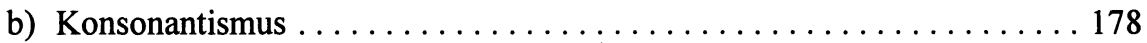

c) Die Betonung der deutschen Ortsnamen $\ldots \ldots \ldots \ldots \ldots \ldots \ldots \ldots \ldots$

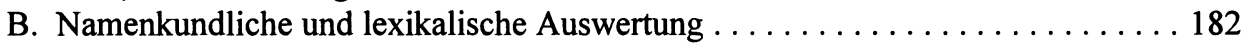

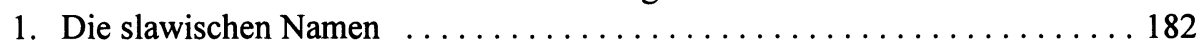

a) Die Bildung und Klassifizierung der slawischen Ortsnamen . . . . . . 182

b) Der in den Namen enthaltene altpolabische Wortschatz . . . . . . . . . . 192

c) Die in den Ortsnamen enthaltenen slawischen Personennamen . . . . . . 195

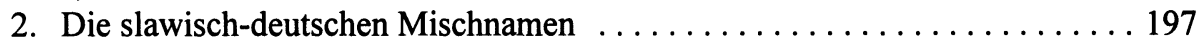

a) Zur Problematik der Mischnamen . . . . . . . . . . . . . . . . . 197

b) Die in den Mischnamen enthaltenen slawischen Personennamen . . . . 198 und die zu ihrer Bildung verwendeten deutschen Grundwörter

3. Die deutschen Namen . . . . . . . . . . . . . . . . . . . . . . . 199

a) Die Bildung der deutschen Ortsnamen $\ldots \ldots \ldots \ldots \ldots \ldots . \ldots \ldots 9 . \ldots \ldots$

b) Die zur Namenbildung verwendeten Grundwörter . . . . . . . . . 202

c) Der in den deutschen Namen enthaltene Wortschatz . . . . . . . . . 211 
d) Die in den Ortsnamen enthaltenen deutschen Personennamen . . . . . . 212

4. Sonstige Erscheinungen, die bei den Ortsnamen unabhängig . . . . . . 213 von ihrer sprachlichen Herkunft vorkommen

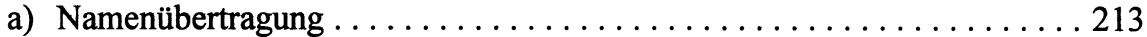

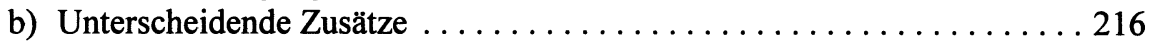

c) Umbenennungen und Namenwechsel . . . . . . . . . . . . 218

d) Das Geschlecht der Namen und der Gebrauch des Artikels . . . . . . . . . . 219

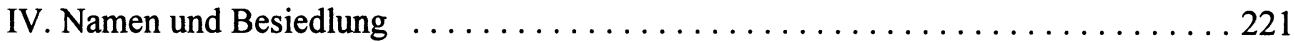

1. Siedlungsgeschichtliche Aspekte der Namen $\ldots \ldots \ldots \ldots \ldots \ldots \ldots \ldots 221$

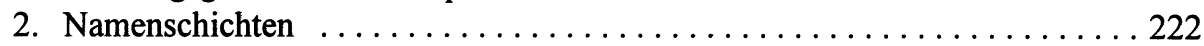

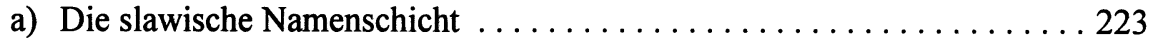

b) Die Namen der feudalen deutschen Siedlung . . . . . . . . . . . . . . 229

c) Die jüngere deutsche Namenschicht $\ldots \ldots \ldots \ldots \ldots \ldots \ldots \ldots \ldots 232$

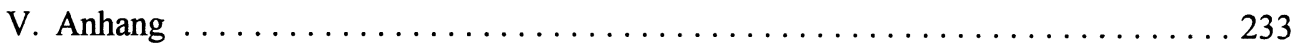

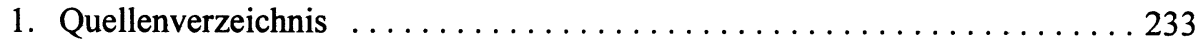

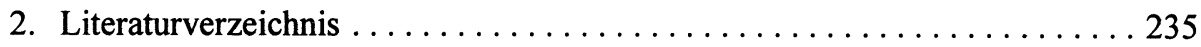

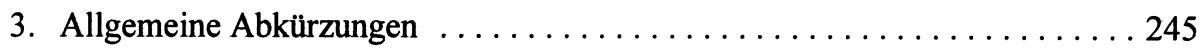

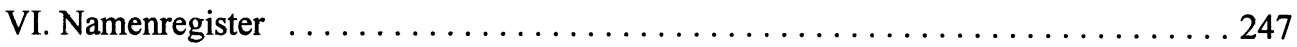

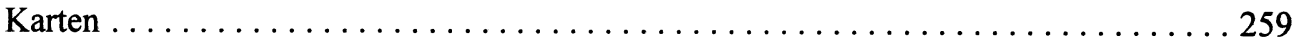

